\title{
Flow statistics in plate and shell heat exchangers measured with PTV
}

Citation for published version (APA):

Beckedorff, L., Nieuwenhuizen, R., Bolwerk, T. M. A. J., Monteiro, A. S., de Paiva, K. V., Kuerten, J. G. M., van der Geld, C. W. M., \& Oliveira, J. L. G. (2019). Flow statistics in plate and shell heat exchangers measured with PTV. International Journal of Heat and Fluid Flow, 79, [108461].

https://doi.org/10.1016/j.ijheatfluidflow.2019.108461

\section{Document license:}

TAVERNE

DOI:

10.1016/j.jijheatfluidflow.2019.108461

Document status and date:

Published: 01/10/2019

\section{Document Version:}

Publisher's PDF, also known as Version of Record (includes final page, issue and volume numbers)

\section{Please check the document version of this publication:}

- A submitted manuscript is the version of the article upon submission and before peer-review. There can be important differences between the submitted version and the official published version of record. People interested in the research are advised to contact the author for the final version of the publication, or visit the $\mathrm{DOI}$ to the publisher's website.

- The final author version and the galley proof are versions of the publication after peer review.

- The final published version features the final layout of the paper including the volume, issue and page numbers.

Link to publication

\section{General rights}

Copyright and moral rights for the publications made accessible in the public portal are retained by the authors and/or other copyright owners and it is a condition of accessing publications that users recognise and abide by the legal requirements associated with these rights.

- Users may download and print one copy of any publication from the public portal for the purpose of private study or research.

- You may not further distribute the material or use it for any profit-making activity or commercial gain

- You may freely distribute the URL identifying the publication in the public portal.

If the publication is distributed under the terms of Article 25fa of the Dutch Copyright Act, indicated by the "Taverne" license above, please follow below link for the End User Agreement:

www.tue.nl/taverne

Take down policy

If you believe that this document breaches copyright please contact us at:

openaccess@tue.nl

providing details and we will investigate your claim. 


\title{
Flow statistics in plate and shell heat exchangers measured with PTV
}

\author{
L. Beckedorff ${ }^{\mathrm{a}}$, R. Nieuwenhuizen ${ }^{\mathrm{b}}$, T.M.A.J. Bolwerk ${ }^{\mathrm{b}}$, A.S. Monteiro ${ }^{\mathrm{c}}$, K.V. de Paiva ${ }^{\mathrm{a}}$, \\ J.G.M. Kuerten ${ }^{\mathrm{b}}$, C.W.M. van der Geld ${ }^{\mathrm{d}}$, J.L.G. Oliveira, ${ }^{\mathrm{a}, *}$ \\ ${ }^{a}$ Department of Mobility Engineering, Federal University of Santa Catarina, Joinville, SC, 89218-035, Brazil \\ ${ }^{\mathrm{b}}$ Department of Mechanical Engineering, Eindhoven University of Technology, P.O. Box 513, 5600 MB Eindhoven, the Netherlands \\ ${ }^{\mathrm{c}}$ CENPES, Petrobras, Cidade Universitária, Rio de Janeiro -RJ, 21941-970, Brazil \\ ${ }^{\mathrm{d}}$ Department of Chemical Engineering and Chemistry, Eindhoven University of Technology, P.O. Box 513, 5600 MB Eindhoven, the Netherlands
}

\section{A R T I C L E I N F O}

\section{Keywords:}

PSHE

PTV

Friction factor

Nusselt number

Shear stress

\begin{abstract}
A B S T R A C T
Particle tracking velocimetry (PTV) measurements have provided inner flow features within plate and shell heat exchangers (PSHE). Measurements have been performed at Reynolds number 3450, based on the bulk velocity and the PSHE geometry at the channel mid-section. Particle trajectories have been measured. Organized flow features prevail in the channel inlet, whereas a highly turbulent flow field occurs at the channel outlet. A recirculation zone characterizes the turbulent flow field at the outlet. Gravity has been shown not to affect flow and heat transfer at this Reynolds number. The mean velocity profile is non-uniform at a given channel cross section. Friction factors developed for Plate Heat Exchanger (PHE) applied to the PSHE geometry with the bulk velocity at the channel mid-plane were found appropriate for design purposes. Furthermore, friction factor, Nusselt number and forces due to shear stresses were locally estimated for the whole channel area. Potential break-down locations have been identified.
\end{abstract}

\section{Introduction}

Plate and Shell Heat Exchangers (PSHE) consist of a fully welded plate pack enclosed within a cylindrical shell. They contain chevrontype plates similar to the ones found in classical Plate Heat Exchangers (PHE) in order to provide high-thermal performance and compactness, Arsenyeva et al. (2016). Unlike conventional PHE structures, the shell structure and laser welding treatment of PSHEs allow operation under severe pressure and temperature conditions. Temperature and pressure levels up to $900{ }^{\circ} \mathrm{C}$ and $20 \mathrm{MPa}$ have been observed, Klemes et al. (2015). Applications range from nuclear power plants to crude oil distillation units, Freire and de Andrade (2014). Considering the advantages of heat transfer and extended operating conditions, PSHEs can replace typical PHEs, Lim et al. (2018).

PSHE heat transfer and hydrodynamic characteristics have been predicted by adapted existing PHE correlations to the use of chevrontype plates. However, the circular plate of the PSHE geometry modifies the flow field, despite the similar corrugated plate geometry. Therefore, further research is essential to improve design of these heat exchangers. In this work the effect of the PSHE plate geometry on pressure drop, heat transfer and forces acting on a typical plate will be investigated with the aid of Particle Tracking Velocimetry (PTV).
The choice for PTV instead of PIV is justified by the acquisition of simultaneous particle information at different locations across a large measurement volume and the inhomogeneous nature of PSHE channel flow, see Oliveira et al. (2017, 2013, 2015). The applied concentration of particles is low to avoid significant dissipation effects and, therefore, avoid any flow field modification induced by particles.

The main bulk flow characteristics are evaluated in a PSHE channel with a $15{ }^{\circ}$ Chevron angle, which is typical for high-pressure drop channels. To ensure optical accessibility, the measurement section consists of two narrow acrylic cylinders, machined to match the PSHE channel geometry. The Reynolds number, based on the bulk velocity and the PSHE geometry at the channel mid-section, equals 3450. This value is representative for oil plant operation and indicates that the flow is turbulent, but flow features will be checked in the present study.

The structure of the paper is as follows. In Section II, the materials and methods used in the paper are shown, including the test rig, specifications of the test section, applied particles, calibration unit, the PTV procedure for identification of individual particle trajectories and the analysis method. In Section III, results are presented for the whole channel, including channel inlet and outlet. They comprise particle trajectories, mean velocities, and estimations for local friction factors, Nusselt numbers and forces due to shear stresses. Section IV presents a

\footnotetext{
* Corresponding author.

E-mail address: jorge.goes@t2f.ufsc.br (J.L.G. Oliveira).
} 
discussion regarding the validation of the method. Finally, conclusions are presented in Sections V.

\section{Materials and methods}

\subsection{Flow characterization}

Significant temperature gradients can yield natural convection effects that may affect the PSHE flow field and therefore the heat transfer performance. The flow situations encountered in practice need to be categorized with the aid of a Grashof number, Gr. A Grashof number can be defined with a typical temperature difference, $\Delta T$, attained with a PSHE characteristic length scale, $L_{v}$, the vertical length between the inlet and outlet PSHE channel centers. Let $v_{\mathrm{B}}$ be the bulk velocity along the vertical direction at the channel mid-plane, $\nu$ the kinematic viscosity, $\boldsymbol{g}$ the gravitational acceleration and $\beta$ the coefficient of thermal expansion. If dissipation is discarded, the typical velocity induced by buoyancy in the $y$-direction over height $L_{v}$ is given by $v_{t b}=\left(g \beta \Delta T L_{v}\right)^{1 /}$ ${ }^{2}$, see de Boer et al. (2017). If this velocity is related to the bulk velocity $v_{\mathrm{B}}$, the ratio of Grashof to Reynolds number squared is obtained:

$G r / R e^{2}=\mathbf{g} \beta \Delta T L_{v} / v_{B}^{2}$

If this ratio exceeds one, natural convection effects are dominant. If it is less than one forced convection effects prevail; see Cengel (2002). By taking fluid properties $(\beta)$ for typical working fluids (water, crude oil and emulsions), heat exchanger dimensions $\left(L_{v} \sim 1 \mathrm{~m}\right)$, operating conditions $\left(10{ }^{\circ} \mathrm{C}<\Delta T<40{ }^{\circ} \mathrm{C}\right)$ and bulk velocities $\left(0.05 \mathrm{~m} / \mathrm{s}<v_{\mathrm{B}}<\right.$ $2 \mathrm{~m} / \mathrm{s}$ ), one can find PSHE channel flow situations where both effects may occur. When the natural convection influence is important, higher temperatures at the PSHE upper region would promote flow stratification. Since adiabatic experiments were conducted, the present experiments are categorized as forced convection dominated. Gravity does not matter and upward and downward flows are expected to yield similar flow fields, but this is something that is validated in the present study.

\subsection{Test rig}

PSHE channel flows have been created in a water loop driven by a centrifugal pump. The in-line $2 \mathrm{~kW}$ pump, type BC-91 S/T from Schneider Motobombas, allows for a maximum mass flow rate of $2.5 \mathrm{~kg} / \mathrm{s}$. A frequency controller and process valves permit fine-tuning of the Reynolds number by adjusting the mass flow rate of the flow in the measurement section. Upward and downward flows have been tested to confirm that gravity would not affect PSHE flows with ratios of Grashof to Reynolds number squared less than one. Water flows through the test section after passing a straight tube with a length of 40 pipe diameters and after passing, subsequently, a mesh flow conditioner. Therefore, the flow is nearly developed at the test section entrance, based on the entrance length correlation of Laws et al. (1987) and prescriptions of the VDI-map.

The mass flow rate is measured by means of a Rosemount $8700 \mathrm{M}$ magnetic flowmeter, whose inaccuracy is less than $0.5 \%$ of the registered flow rate. A water reservoir contains about $0.35 \mathrm{~m}^{3}$ of water. This size facilitates water temperature stabilization and hence Reynolds number control. Thermocouples monitor water temperature in the flow loop.

Fig. 1 presents details of the setup. The grey parts of (Fig. 1a) are related to facilities to create a three-phase (oil, water and air) flow. Figure Fig. 1b shows a photograph of the set-up built at the Thermal Fluid Flow Group - T2F laboratory, Universidade Federal de Santa Catarina, Brazil.

The measurement section will be described below. Water leaves and enters the section through pipes with $56 \mathrm{~mm}$ inner diameter. Pressure taps were installed just upstream and downstream of the test section.
An Omega differential pressure transducer, type PX409 Series Differential Pressure, monitors the pressure drop over the test section. The transducer uncertainty is $0.5 \%$ of the registered pressure drop.

Measurements have also been performed with a smooth titanium PSHE test section for comparison.

\subsection{Test section}

Two circular acrylic disks with a thickness of $1.5 \mathrm{~cm}$ were machined to make up a typical PSHE channel when pressed together, see Fig. 2. Fig. 2a presents the main dimensions of the channel frontal view of one plate. The external diameter, $L_{w}$, is $295 \mathrm{~mm}$, whereas the length between the inlet and outlet centers, $L_{v}$, is $210 \mathrm{~mm}$. A Cartesian coordinate system with its origin in the frontal view center is taken with the $y$-axis joining the centers and with gravity anti-parallel to the $y$-axis. The Chevron angle, $\beta$, denotes the angle between the flow channel main direction and the $x$-axis; $\beta$ is chosen as $15^{\circ}$, which is typical of high pressure drop channels (indicated by the letter " $\mathrm{H}$ ").

Fig. $2 \mathrm{~b}$ provides details of the cross-section modifications along the $y$-axis. $P_{c}$ is the corrugation pitch, and $b$, the corrugation amplitude. Here, $P_{c}$ is $33.7 \mathrm{~mm}$ and $b$ is $1.95 \mathrm{~mm}$. Fig. $2 \mathrm{c}$ presents the cross section variation in $y$-direction between the channel inlet and outlet. The cross section, $A_{\text {sec }}$, increases towards the channel midsection $(y=0)$.

Fig. $2 \mathrm{~d}$ and e present the channel height, $t$, variation in $x$-direction (d) and $y$-direction (e); see dash-dotted lines in Fig. 2a.

Reynolds numbers, based on the bulk velocity at the channel center point $(x=y=0), v_{\mathrm{B}}$, and channel maximum height, $t_{\max }$, can be as high as 9500 . For a flow rate of $0.0025 \mathrm{~m}^{3} / \mathrm{s}$, the Reynolds number based on the average cross section area of $535 \mathrm{~mm}^{2}$ and a length scale equal to the average height, $t_{\text {ave }}=1.95 \mathrm{~mm}$, is 10,100 .

\subsection{Tracer particles}

Gravel seeding particles with a diameter of $0.15 \mathrm{~mm}$ and a mass density of $1.6 \mathrm{~g} / \mathrm{cm}^{3}$ have been added to the water as flow tracers. In order to analyze how particles interact with the fluid, time and length scales of particles, $\tau_{p}$ and $l_{p}$, respectively, are compared to the smallest fluid scales, $\tau_{f}$ and $l_{f}$. The subscripts $p$ and $f$ denote particle and fluid. The relaxation time for particles in stationary flow is shown by Albrecht et al. (2003) to be:

$\tau_{p}=\left(d_{p}^{2} \rho_{p} / 18 \mu\right)\left(1+0.5 \rho_{f} / \rho_{p}\right)$

where $d_{p}$ is the particle diameter, $\rho$ the mass density and $\mu$ the dynamic viscosity. A relaxation time of $\tau_{p} \approx 2.6 \mathrm{~ms}$ is obtained for the gravel particles.

Assessment of dissipative fluid time and length scales are performed with viscous wall units, $\tau_{k}=\nu / u_{\tau}^{2}$ and $l_{k}=\nu / u_{\tau}$, respectively, where $\nu$ is the kinematic viscosity and $u_{\tau}$, the wall shear velocity. The latter is defined as $u_{\tau}=\left(\tau_{w} / \rho_{f}\right)^{1 / 2}$ where $\tau_{w}$ is the wall shear stress. An order-ofmagnitude estimation can be made by using $\tau_{w}=\mu v_{\mathrm{B}} / t_{\text {ave }}$. With $v_{\mathrm{B}}$ equal to $0.84 \mathrm{~m} / \mathrm{s}$ and for water flows at atmospheric conditions, $\nu \approx$ $10^{-6} \mathrm{~m}^{2} \mathrm{~s}^{-1}$, estimations of $\tau_{w}, u_{v} l_{k}$, and $\tau_{k}$ are, respectively: $0.39 \mathrm{~Pa}$, $19.75 \mathrm{~mm} / \mathrm{s}, 0.0457 \mathrm{~mm}$ and $2.3 \mathrm{~ms}$.

With the computations above, particle and fluid time and length scales have been compared. The Stokes number, $\mathrm{St}=\tau_{p} / \tau_{k}$, is 1.13 , while the ratio of particle diameter to the fluid length scale, $d_{p} / l_{k}$, is 3.28 .

Gravitational effects on the applied particles are evaluated by comparing the terminal velocity of gravel particles, $U_{T V}$, to the bulk flow velocity at the channel mid-plane, $v_{\mathrm{B}}$. The terminal velocity is given by Eq. (3):

$U_{T V}=\left\{\left(4\left(\rho_{p}-\rho_{f}\right) d_{p} \mathbf{g}\right) /\left(3 C_{D} \rho_{f}\right)\right\}^{1 / 2}$

where $C_{D}$ the drag coefficient. The latter is a function of the particle Reynolds number, $R e_{p}=d_{p} \mid\left(U_{T V} \mid / \nu\right.$, which is based on the particle 




a



b

Fig. 1. Multiphase flow experimental setup: (a) schematics and (b) photograph. Only the water circuit (colorful graphic in "a") was applied for experiments.

diameter and the terminal velocity. For $0.01<R e_{p}<20$, Clift et al. (1978) defined a correlation for $C_{D}$ as given by Eq. (4):

$C_{D}=\left(24 / R e_{p}\right)\left(1+0.131 R e_{p}^{0.82-0.05 \log _{10} R e}{ }_{p}\right)$

A numerical value for $U_{T V}$ can be obtained by an iterative calculation using Eqs. (3) and (4) and $R e_{p}$. An estimate of $10.9 \mathrm{~mm} / \mathrm{s}$ was obtained for $U_{T V}$, while $v_{\mathrm{B}}$ is approximately $840 \mathrm{~mm} / \mathrm{s}$ for the present case. Since $v_{\mathrm{B}}>>U_{T V}$, gravitational effects are insignificant for the tracers.

The effect of flow acceleration on particles also requires assessment. Its relevance to particle motion can be revealed by comparing the ratio of pressure gradient force, including the added mass force, to the buoyancy force,

$\left[3 / 2 V_{p} \rho_{f} D U_{L} / D t\right] /\left[V_{p}\left(\rho_{p}-\rho_{f}\right) \mathbf{g}\right]$

where $V_{p}$ is the particle volume and $\mathrm{D} U_{\mathrm{L}} / \mathrm{D} t$ the Lagrangian fluid acceleration. The added mass coefficient is taken to be 0.5 . $\mathrm{D} U_{\mathrm{L}} / \mathrm{D} t$ is roughly approximated by the bulk velocity at the channel mid-section times its change along the vertical direction, $v_{\mathrm{B}} \partial v_{\mathrm{B}} / \partial y$. While $v_{\mathrm{B}}$ is $0.84 \mathrm{~m} / \mathrm{s}, \partial \nu_{\mathrm{B}} / \partial y$ can be estimated with the aid of Fig. 2c, considering $t_{\text {ave }}$ constant at each cross section $A_{\text {sec }}$. With $\partial v_{\mathrm{B}} / \partial y$ estimated as $0.4 \pi$, the force ratio given by Eq. (5) scales as $1 / 300$. Therefore, flow acceleration on particles can be neglected.
Lastly, it is important to assess the ratio of particle diameter to the PSHE channel depth. Here, $d_{p} / t_{\max }$ is 0.0385 . This value suggests that particle volume is significant and lift forces may affect the particle trajectory. This will therefore be investigated in section III.

\subsection{Optical settings}

One "SpeedSense" camera with 12-bit grayscale CMOS sensor and a resolution of $1280 \times 1280$ pixels has been utilized to capture almost instantaneous 2D particle positions in a circular area with a diameter of $30 \mathrm{~cm}$. The camera operated at $1000 \mathrm{~Hz}$. Recordings are performed until the internal memory of the camera becomes full during approximately $2 \mathrm{~s}$. The above estimate of $\tau_{k}$ at $R e_{b}=3450$ assures high-accuracy tracking with a $1000 \mathrm{~Hz}$ sampling rate.

For the present experiment, the settings can be summarized as: pixel sensor resolution equal to $10 \mu \mathrm{m}^{2}$; focal length of $100 \mathrm{~mm}$; exposure time of $1 \mathrm{~ms}$; distance from the lens to the object of roughly $1 \mathrm{~m}$.

\subsection{Particle tracking algorithm}

The commercial PTV imaging code from La Vision GmbH, named Davis, has been used to obtain tracer trajectories. Algorithm details of the Davis PTV tracking code can be found in Maas (1996) and 


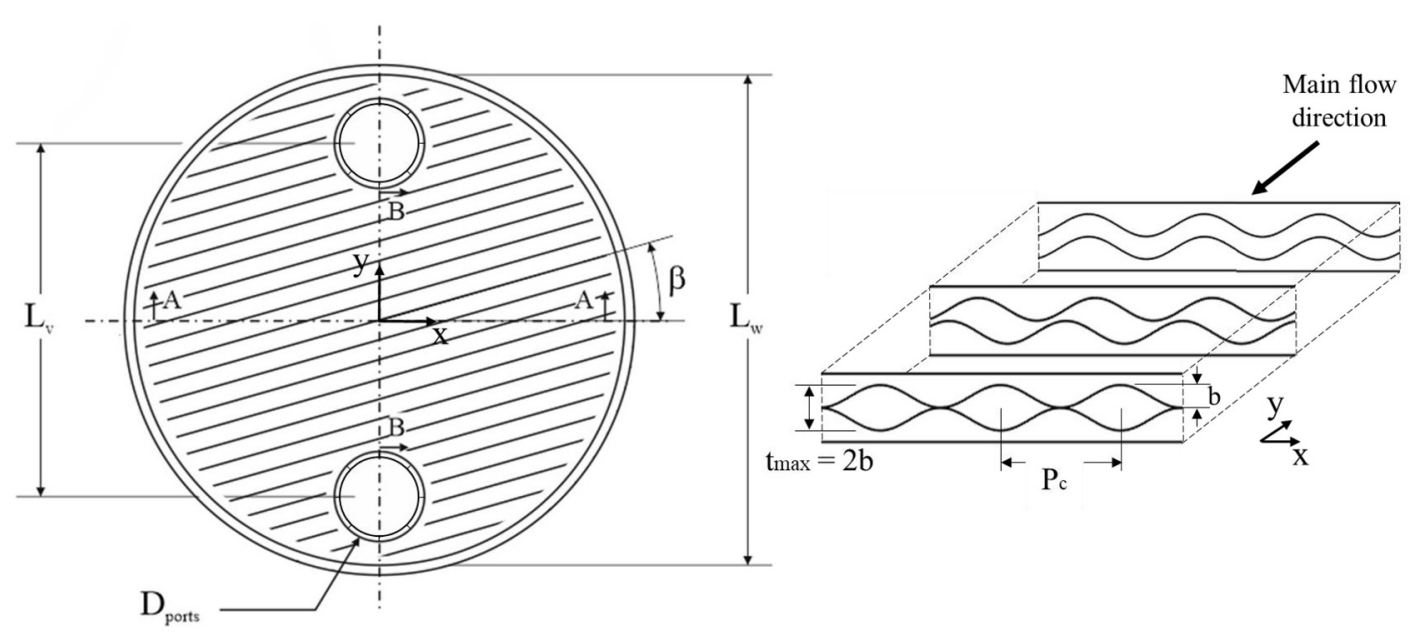

a

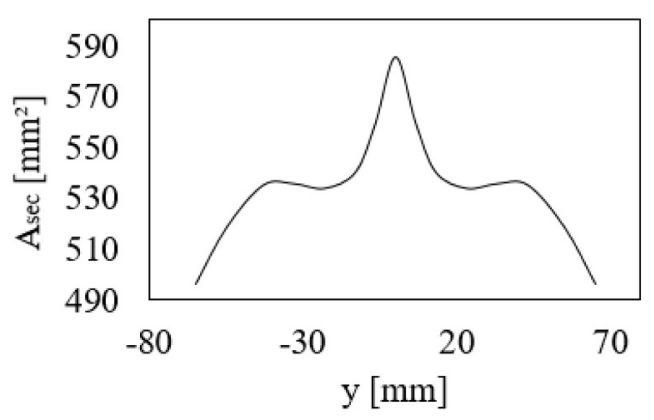

$\mathrm{c}$ b

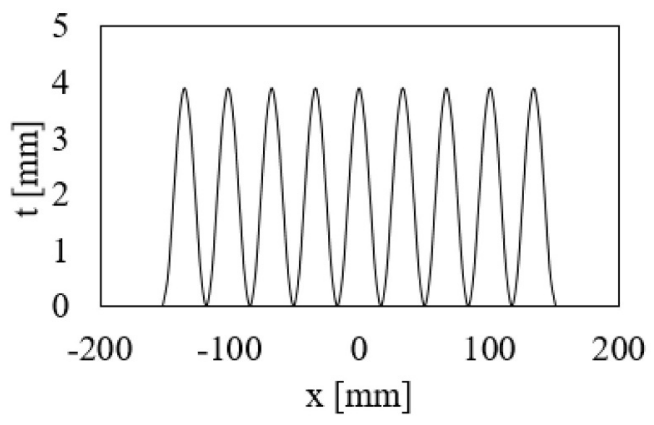

d

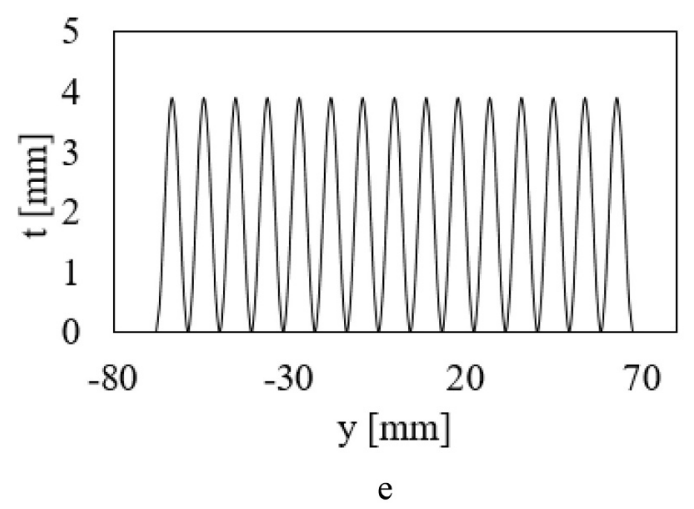


direction, and channel depth variation along the horizontal section A-A (d) and vertical section B-B (e).

Dracos (1996). The PTV procedure for identification of individual particle trajectories resembles the one applied by Oliveira et al. (2013) for pipe flow. Calibration and flow measurement images are processed in order to output files which contain time reference and spatial positions of individual particle trajectories.

To correlate the camera pixel information to world coordinates, a calibration plate, made of plastic and with $0.5 \mathrm{~mm}$ thickness, has been used. The calibration recording is registered in one position only and, therefore, the trajectory analysis is restricted to the frontal view plane of the channel.

The centers of the circular voids in the plate are equidistant in horizontal and vertical direction: $\Delta x, \Delta y=20 \mathrm{~mm}$; see Figs. 3a-3c, which provide a view of the calibration plate. With the information of the pixel size of the cameras, $10 \mu \mathrm{m}^{2}$, and the diameter of the circular voids, $\Phi=1 \mathrm{~mm}$, 3rd order polynomials relate the pixel information to the physical dimensions of the calibration plate. The circular void diameters are accurate within $0.5 \mu \mathrm{m}$. The relative position of two neighboring grid points is accurate within $0.1 \mu \mathrm{m}$. Linear interpolations and extrapolations of the generated polynomials are extended to the whole area of measurement. Root-mean-square (RMS) errors of the generated functions are smaller than 0.2 pixel, approximately $2 \mu \mathrm{m}$.

In Figs. 3a - 3e, camera photos show different stages of the PTV procedure. Once the calibration procedure is completed (Figs. 3a-3c), the calibration plate is removed and flow measurements are recorded (Fig. 3d). Built-in imaging filters improve the contrast between particles and background. The 2D determination of the center of a tracer in the camera plane is done by a Gaussian fit and cataloged only if its intensity threshold is larger than a certain default value. The 2D particle detection is then initiated and its procedure is similar to the one applied by Oliveira et al. (2015). The polynomials created during the calibration 


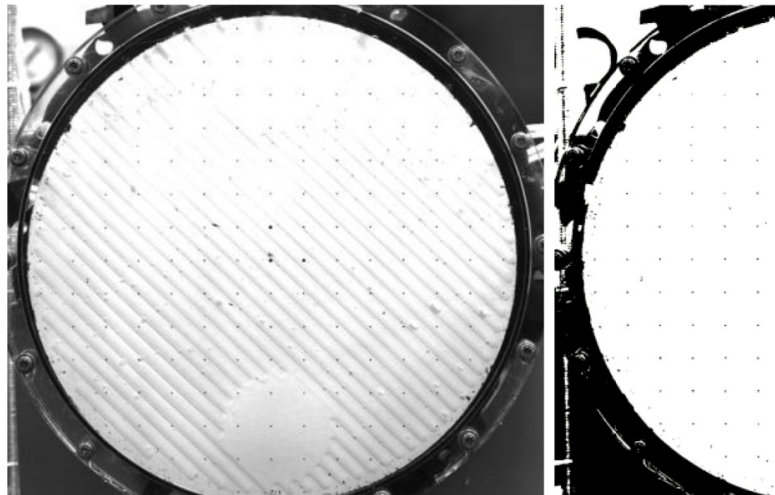

a

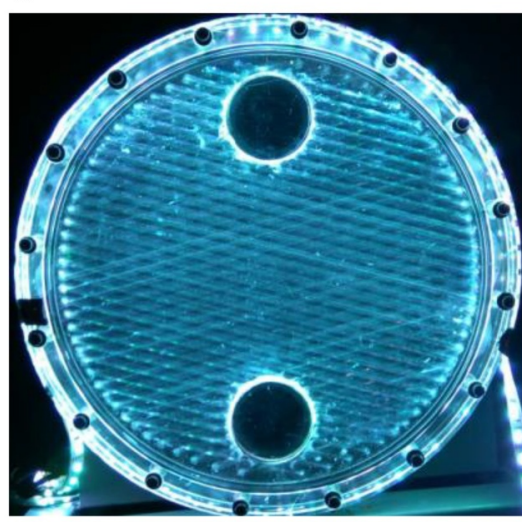

d

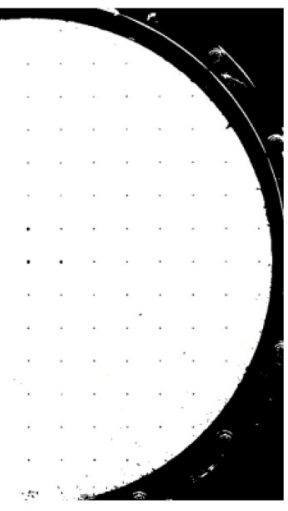

b

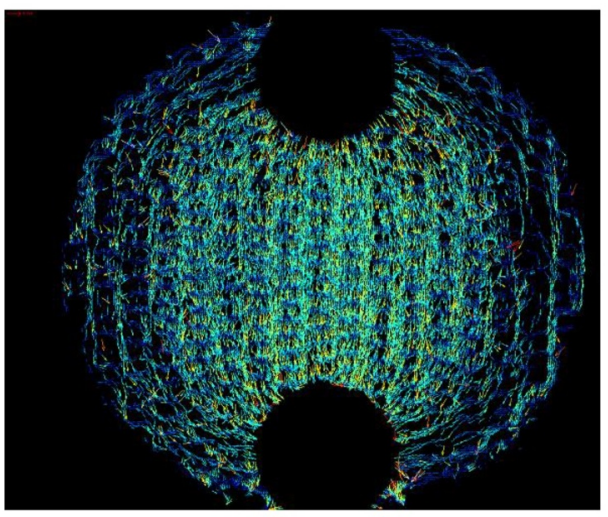

e

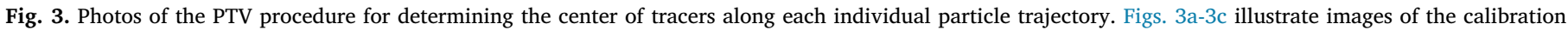

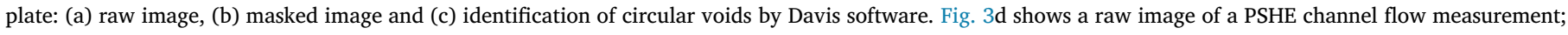
Fig. 3e shows the merging of individual tracer trajectories in a finite time window.

stage are used to determine the spatial Cartesian coordinates. Finally, matrices with the spatial coordinates and the time of each particle position successfully identified are exported to the analysis method.

Fig. 3e presents the merging of individual tracer trajectories in a finite time window. The colorful vectors represent the orientation and magnitude of particle velocities. Most of the generated spurious vectors are located in the channel periphery and removed in a way to be described in the next section.

\subsection{Analysis method}

The particle-tracking algorithm yields matrices that contain time reference and spatial positions of particle trajectories from the flow measurement images. Spurious trajectories that are generated during the PTV procedure are discarded in the following way.

Two filters accomplish the removal of unrealistic trajectories: a length filter and a displacement outlier-check at the channel periphery. The length filter eliminates all trajectories of tracers with a number of positions of a particle track that is less than a certain threshold. This procedure has proven to remove unrealistic particle trajectories, for example by elimination of particle trajectories consisting of less than four spatial positions.

There is a higher than average number of measured particle trajectories in grid cells surrounding the channel periphery. The difficulties in measuring particle trajectories in this region are caused by light reflections, which stem from differences in the refractive indices of water, $n \approx 1.33$, and acrylic, $n \approx 1.51$, and the curvature of the acrylic plates; see Fig. 3d. As a result, light reflections resemble flow tracers. However, the reflections are constant in time and by treating trajectories with streamwise velocity equal to zero as spurious vectors, it is possible to remove almost all noise at the channel edges. Moreover, in order to reduce reflections at the test section, a lighting system consisting of LEDs was positioned around the channel periphery, invisible to the fast camera.

After the removal of the spurious particle tracks, differentiation of the validated trajectories with respect to time generates the velocity vectors. The vectors are then gathered in discrete bins; see Fig. 4. No biasing correction has been applied in the analysis of the experimental data; this is in contrast to the procedures followed by Walpot et al. (2006) and Oliveira et al. (2015), for example. A camera frequency of $1000 \mathrm{~Hz}$ sufficed to obtain negligible bias at $R e_{b}=3450$.

In order to facilitate flow analysis in the channel inlet and outlet, the spatial positions are converted from Cartesian to cylindrical coordinates, with their origins in the inlet and outlet manifold centers. Velocities in the radial direction, $v_{\text {rad }}$, are gathered in bins that form a narrow ring adjacent to the channel inlet and outlet; see Fig. 5.

Particle trajectories have been registered in 20 individual measurement sets of $2 \mathrm{~s}$ each. The differentiation of particle trajectories with respect to time generated approximately $10^{5}$ velocity vectors. The velocity vectors are ensemble-averaged in distinct grid points around the channel inlet, around the channel outlet and in the PSHE channel as presented above.

\section{Results}

Velocity statistics are normalized by the bulk velocity at the channel mid-section (plane $y=0$; see Fig. 2a). The bulk velocity at $y=0$ is chosen as a normalization quantity, because the PSHE channel Reynolds number is typically computed with this reference velocity and, therefore, it can assist in comparisons with other experimental results. 




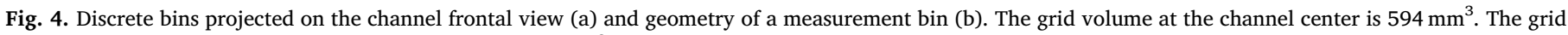
volumes at the channel boundaries range from 74 to $465 \mathrm{~mm}^{3}$.

Throughout this article, the errors of time-averaged values of a measured quantity $x$ are estimated with the aid of the so-called standard error, $\sigma_{m}$. Confidence intervals of $95 \%$ are considered. For a quantity, which is measured $n$ times, with instantaneous results $x_{i}$ and mean $\langle x\rangle$, the standard error is given by:

$\sigma_{m}=\sum_{i=1}^{n}\left[\left(x_{i}-\langle x\rangle\right)^{2} / n(n-1)\right]^{0.5}$

with $x_{i}$ the average over a single measurement set and $n$ the total number of measurement sets: 20 .

\subsection{Particle trajectories}

Fig. 6 presents samples of particle trajectories obtained with PTV in the whole channel front view (Fig. 6a), in the channel inlet (Fig. 6b) and in the channel outlet (Fig. 6c) for upward flow. Each figure represents the merging of trajectories for several flow images.

Fig. 6a shows particle trajectories that resemble longitudinal wavy flow (helical type) as presented by Focke and Knibbe (1986) for channels in Plate Heat Exchangers with elevated pressure drops. Note that particles hardly follow the corrugation angle direction $\left(15^{\circ}\right.$ with the horizontal). According to Grabenstein et al. (2017), the PHE Reynolds number and the corrugation angle affect the flow structures in the
PHE channel. At high Reynolds numbers or low corrugation angles (here, the angle formed with the axis perpendicular to the main flow direction), the fluid takes the shortest way down the channel. For low Reynolds numbers or high corrugation angles, the fluid mainly travels through the Chevron angle direction.

Differences in flow trajectories in the PSHE channel inlet and outlet are discussed with the aid of Fig. $6 \mathrm{~b}$ and c. While flow trajectories enter the PSHE channel in well-behaved streamlines, high turbulence levels and recirculation characterize the flow in the PSHE channel outlet. Recirculation zone occurs in the channel outlet (Fig. 6c). These results can be explained in the following way.

In the channel inlet, the annular cross section surrounding the PSHE entrance is smaller than the entry pipe cross section. Therefore, the flow accelerates in the PSHE entrance, while the static pressure reduces. The flow leaves the entry pipe with positive radial velocity towards the PSHE channel. At the PSHE channel outlet, the opposite occurs: the flow decelerates, while the static pressure increases. The increasing pressure promotes boundary layer separation near the channel outlet, resulting in recirculation zones.

\subsection{Velocity field}

Fig. 7 shows the mean vertical and lateral velocity components in the PSHE.



a

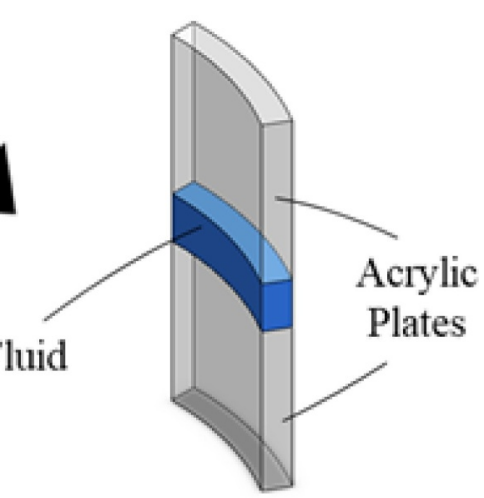

b

Fig. 5. Discrete bins projected in the frontal view of the channel inlet or outlet (a) and geometry of a measurement bin at the same positions (b). The grid volume is $502 \mathrm{~mm}^{3}$. 




a



b

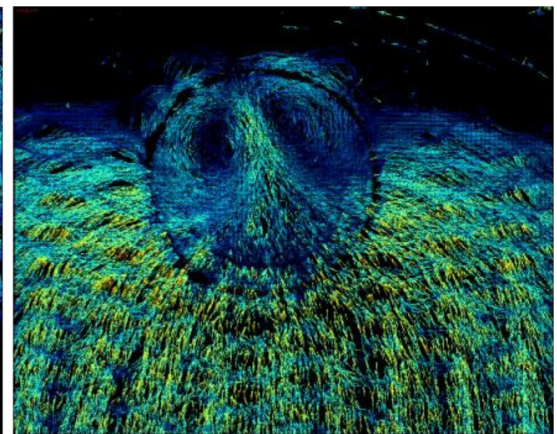

c

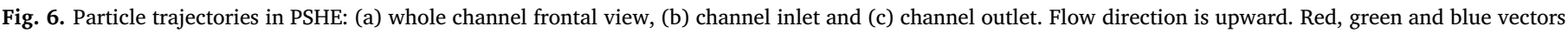
indicate largest, intermediate and smallest particle velocity in that order.

The differences between the entrance and exit velocity fields (see Fig. $6 \mathrm{~b}$ and $\mathrm{c}$ in the previous section) hardly affect the symmetry of the mean velocity in the $y$-direction. Note that the streamwise velocity is nearly symmetric with respect to the mid-plane $y=0$; see Fig. $7 \mathrm{a}$, while the lateral bulk velocity is anti-symmetric related to both the plane $y=0$ and the plane $x=0$; see Fig. $7 \mathrm{~b}$.

At a given cross section between the exit and entrance manifolds, the mean velocity is higher in the central area $(-0.07 m<y<0.07 \mathrm{~m}$; $0.07 m<x<0.07 \mathrm{~m}$ ), see the blue region in Fig. 7a. The flow resistance is smaller due to the shorter exit length; compare the length of particle trajectories in the central region, $x \sim 0$, and away from the central area, $x \neq 0$, in Fig. 6 a.

Fig. 8 shows profiles of the mean streamwise (Fig. 8a) and lateral (Fig. 8b) velocity components. In order to demonstrate the symmetry of the mean streamwise velocity field (and anti-symmetry of the lateral velocity profile), three $y$-positions in the channel coordinate system, $-0.06,0$ and 0.06 , are chosen. The velocity is made non-dimensional with the bulk velocity, $v_{\mathrm{B}}$.

Note in Fig. 8a that the streamwise profiles match within experimental uncertainty at $y$-planes -0.06 and 0.06 . Error-bars give an indication of the variation of velocity over the height. The peak velocity in the center point $(y=0 ; x=0)$ is $1.5 v_{\mathrm{B}}$, and slightly lower than at positions $(y= \pm 0.06 ; x=0)$ due to mass conservation. At these positions, the maximum velocity reaches 1.7 of the bulk velocity. The lateral velocity changes direction in the Cartesian coordinate system at the same cross section. It is also approximately anti-symmetric to the plane $x=0$; see Fig $8 \mathrm{~b}$.

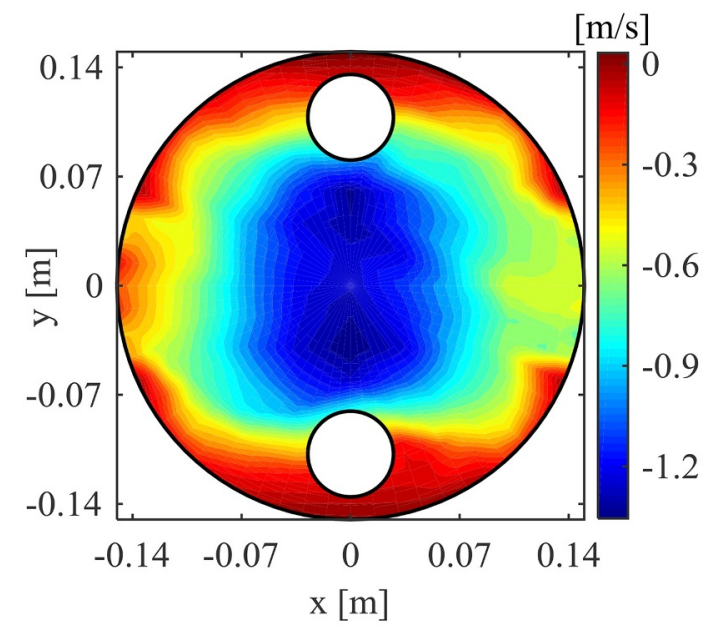

a
Fig. 9 shows the mean velocity at the inlet and exit channel circumference. For convenience, cylindrical coordinate systems with their origins at the center of the inlet and outlet are used, as defined in Fig. 5. Results are plotted in clockwise direction for the outlet and in counter clockwise direction for the inlet. The radial velocity, $v_{\text {rad }}$, is made nondimensional with $v_{\mathrm{B}}$.

Variations in the mean radial velocity are more intense at the PSHE outlet than at the inlet. The flow recirculation, which characterizes the PSHE channel outlet, affects the radial velocity distribution. Note that the radial velocity can be as high as $1.20 v_{\mathrm{B}}$, whereas it is below $0.65 v_{\mathrm{B}}$ at the PSHE entrance. At the outlet segment $0<\theta<\pi$, the mean radial velocity component is close to zero because of the recirculation zone. Due to mass conservation, the radial velocity is larger at the outlet segment $\pi<\theta<2 \pi$. At the inlet segment $0<\theta<\pi$, the flow resistance is high due to the wall presence at the PSHE circumference and therefore the radial velocity is lower than at the inlet segment $\pi<\theta<$ $2 \pi$.

\subsection{Friction factor, Reynolds and Nusselt numbers}

The velocity field as presented in the previous section affects heat transfer and pressure drop across the PSHE channel. The local Reynolds number, $R e(x, y)$, friction factor, $f(x, y)$, and Nusselt number, $N u(x, y)$, are calculated as functions of the local convective velocity, $v(x, y)$. It is assumed that typical Nusselt and friction factor correlations for the PHE can be locally applied in a given PSHE channel. The magnitude of the velocity vector is found from the horizontal and vertical mean velocity

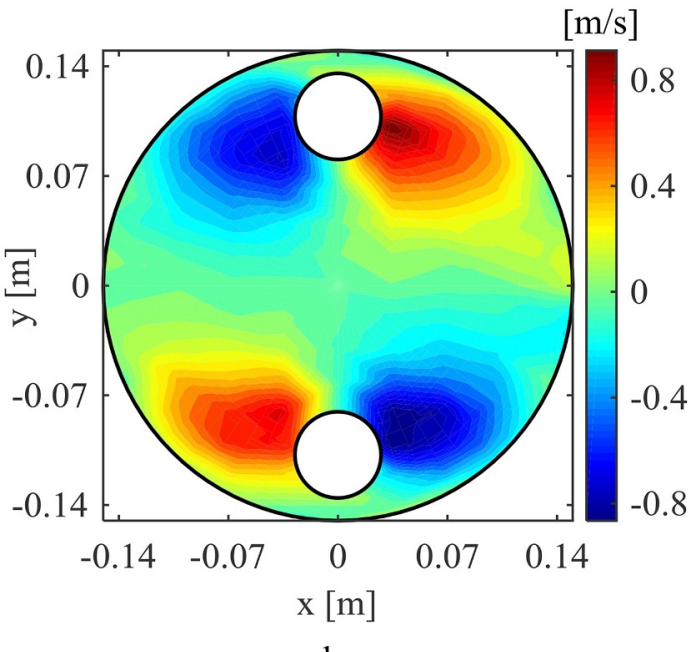

b

Fig. 7. Mean velocity in the PSHE: (a) vertical and (b) horizontal components. Flow direction is downward. 




a

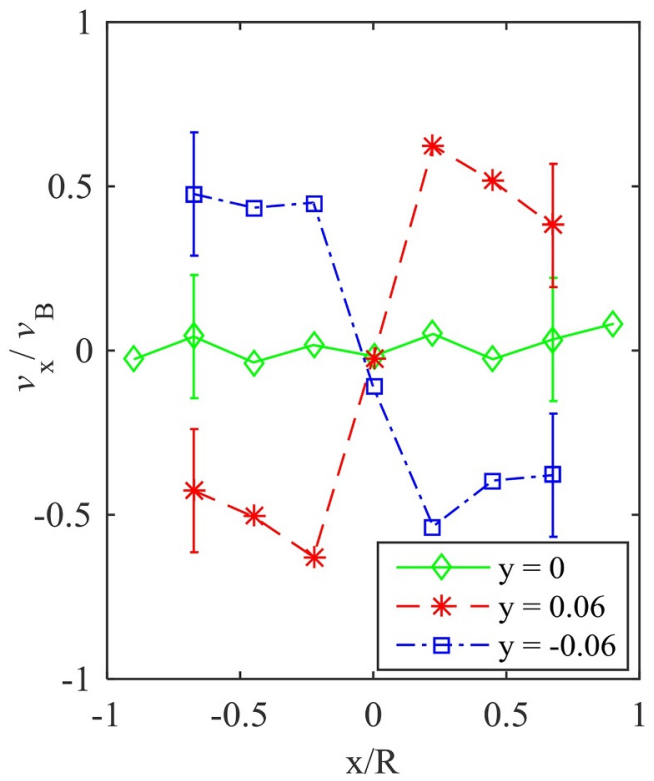

b

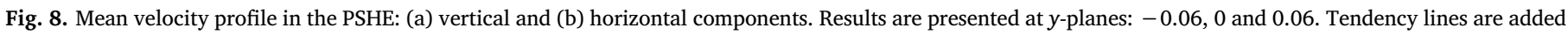
to guide the eye. Flow direction is downward.

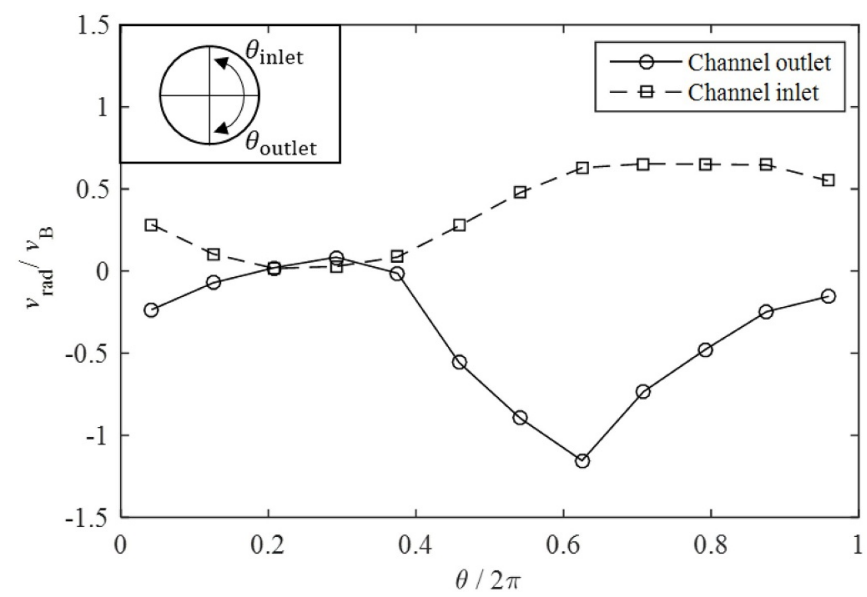

Fig. 9. Mean radial velocity profile at the inlet and exit channel circumference. Tendency lines are added to guide the eye. These results are for downward flow but upward flow gave much similar results.

components as presented in Fig. 7. The local Reynolds number is calculated as:

$\operatorname{Re}(x, y)=\rho|v(x, y)|(2 b / \phi) / \mu$

where $\phi$ is the enlargement factor and $\mu$ the dynamic viscosity.

The correlations presented by Heavner et al. (1993) for friction factor, $f$, and Nusselt number, $N u$, Eqs. (8) and 9, were chosen for this purpose:

$f=0.490 \phi^{1.1814} R e^{-0.1814}$

$N u=0.089 \operatorname{Pr}^{1 / 2}\left(\mu / \mu_{w}\right)^{0.17} \phi^{0.282} \operatorname{Re}^{0.718}$

where $\operatorname{Pr}$ is the Prandtl number and $\mu_{w}$ the dynamic viscosity at the wall. Note that $f(x, y)$ and $N u(x, y)$ depend on the local $R e(x, y)$.

Fig. 10 shows the local PSHE channel Reynolds number (Fig. 10a), friction factor (Fig. 10b) and Nusselt number (Fig. 10c).

The local $\operatorname{Re}(x, y)$ can attain values up to 5250 at $x \approx 0$, whereas it can drop to less than 1000 at the channel boundaries; see Fig. 10a. Nusselt and Reynolds color-maps are qualitatively similar, since $\mathrm{Nu}$ $(x, y)$ is proportional to $R e(x, y)^{0.718}$; see Eq. (9). The local Nusselt number can attain values up to 110 at $x \sim 0$, whereas it can drop to less than 30 at the channel boundaries; see Fig. 10c.

The friction factor map differs from the Reynolds and Nusselt maps. The local friction factor is proportional to $\operatorname{Re}(x, y)^{-0.1814}$; see Eq. (8). In the channel center $[-0.05 m<x<0.05 \mathrm{~m} ;-0.05 m<y<0.05 \mathrm{~m}], f$ $(x, y)$ ranges from 0.12 to 0.16 . Local friction factors increase in the ranges $x<-0.05 \mathrm{~m}$ and $x>0.05 \mathrm{~m}$, since local velocities and Reynolds numbers decrease there.

By averaging local friction factors, Reynolds and Nusselt numbers over the total area, values equal to $0.135,3300$ and 76 , respectively, are obtained. The PSHE Reynolds number computed at the channel mid-plane, 3450 , differs by only $4.3 \%$. The friction factor and Nusselt number calculated from the correlations by Heavner et al. (1993) with the mid-plane PSHE Reynolds number are 0.132 and 80.15 , respectively. The observation that these results are close to the averages of the local values, suggests that local friction factors, Reynolds and Nusselt numbers are representative for the PSHE channel.

\subsection{Shear forces}

The local force due to wall shear stress is estimated on the channel projection in the $x y$-plane in the following way. The magnitude of the local force, $F(x, y)$, is obtained by the product of the local shear stress, $\tau_{w}(x, y)$, and the local area projected in the $x y$-plane, $\mathrm{A}(x, y)$ :

$F(x, y)=\tau_{w}(x, y) \mathrm{A}(x, y)$

where the subscripts $x$ and $y$ stand for a volume grid location. $\mathrm{A}(x, y)$ is typically $785.3 \mathrm{~mm}^{2}$ for only one plate; see Fig. 4 a. $\tau_{w}(x, y)$ is computed with the local Fanning friction factor, $f(x, y)$, and the local bulk velocity, $v(x, y)$, as:

$\tau_{w}(x, y)=1 / 2 f(x, y) \rho|v(x, y)|^{2}$

Note that the pressure loss due to the PSHE converging-diverging channel geometry is not considered.

Fig. 11a shows the local force magnitude projected on the channel frontal area. The local force is calculated with the wetted area. Since the direction of the force changes from point to point in accordance to the mean velocity direction, the local forces are decomposed into 


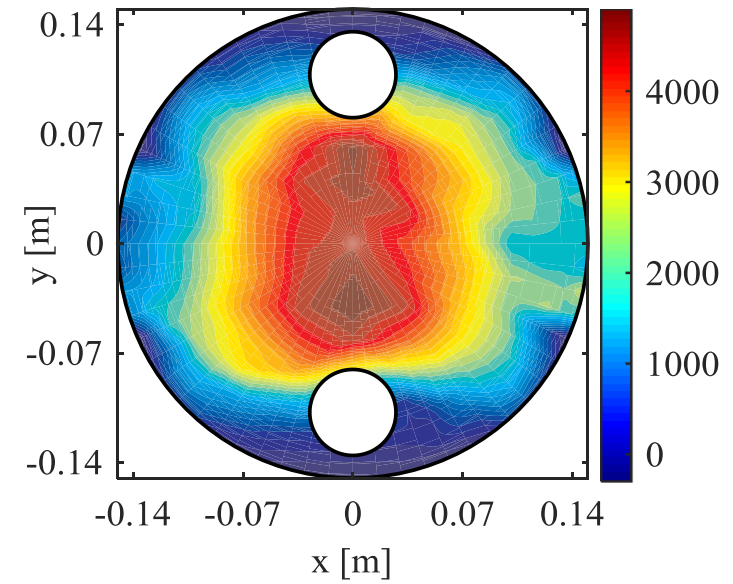

a


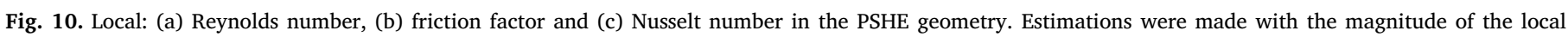
absolute mean velocity in a given grid point.

horizontal and vertical directions and shown in Fig. 11b and c.

Forces in horizontal direction are significant only near the inlet and outlet channels, whereas forces in vertical direction are significant in the central channel area. The force is maximum near the outlet boundary, opposed to the recirculation zone $(0<\theta<\pi)$. Of course, that coincides with the location of the highest mean velocity. Note, however, that fatigue can also affect the welding structure at the channel exit. For upward flows, the bottom edge of the channel $(\pi<\theta$ $<2 \pi$ ) is affected by recirculation zones, although the mean flow velocity is nearly zero.

Due to the symmetry of the flow with respect to plane $x=0$, the integral of the average force in horizontal direction equals zero. At the present flow entrance conditions, integration of the average force in vertical direction results in nearly $18.7 \mathrm{~N}$ for one channel wall. For the pair of plates, the total mean vertical force is $37.4 \mathrm{~N}$. In order to check if the estimates above are appropriate, the total shear stress at the wall is computed by the mean equation of motion:

$\tau_{w}=\left(\Delta P A_{\text {inlet }}\right) / A_{\text {channel }}$

where $A_{\text {inlet }}$ is the channel inlet or outlet area, and $\Delta P$ is the pressure drop over the channel. At the wall, the Reynolds stress is zero, and the wall shear stress is entirely caused by the viscous contribution. For $R e_{b}=3450, \Delta P$ is roughly 0.11 bar. The total shear stress as computed by Eq. (12) differs by only $12 \%$ from the total shear stress found from integrating Eq. (11).

\section{Discussion}

The integration over a given PSHE cross section of the product of the mean axial velocity and the cross-sectional area of each grid cell, $U_{z} A$, gives the mean volumetric flow rate, $Q$. Temperature measurements are used to determine the mass density of water, $\rho$. The product $\rho Q$ provides a measure of the mass flow rate for a selected y-plane. In order to validate the present PTV method, PSHE mass flow rate computations are performed in the channel inlet and outlet, and along the channel cross section. The results agree with the mass flow rates given by the electromagnetic flowmeter within the statistical error as shown below.

Fig. 12 shows the mass flow rate calculated along the PSHE channel cross section with the aid of PTV. A Cartesian coordinate with its origin in the channel front view center is applied; see Fig. 2a. The mass flow rate is determined in 15 cross sections of the channel between the inlet and outlet manifolds: $-0.06<y[\mathrm{~m}]<0.06$.

The mean mass flow rate obtained by the electromagnetic flowmeter is $0.491 \mathrm{~kg} / \mathrm{s}$. Note that the mass flow rates obtained with the PTV method matches the Coriolis measurement within the measurement uncertainty; see error-bars, Eq. (6), in Fig. 12. Confidence intervals of $95 \%$ are considered. The considerable error-bars are due to the experimental 2D-PTV limitations, as it will be explained below. Since mass conservation was achieved throughout the PSHE cross sections, particle migration perpendicularly to the channel wall was not important for bulk flow measurements. 


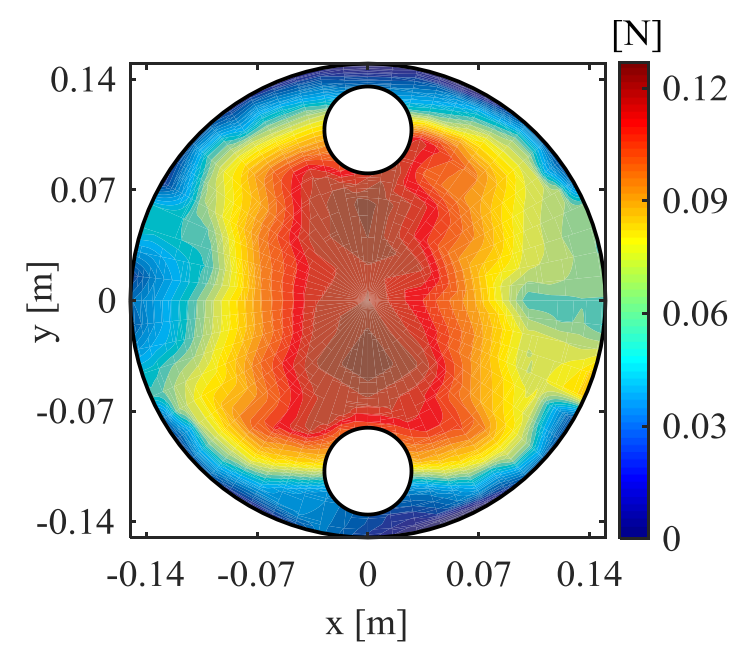

a



b

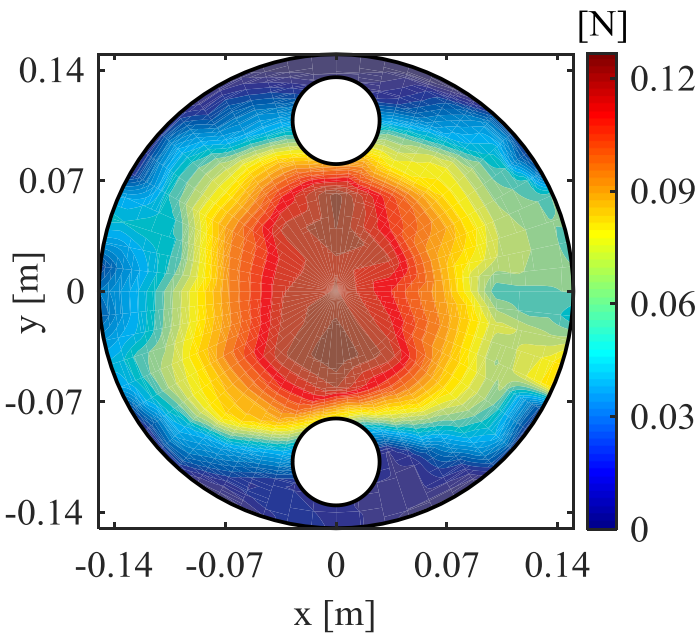

c

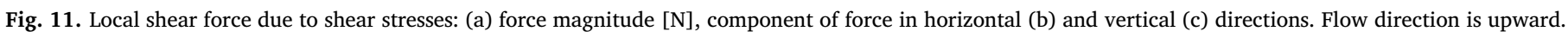



Fig. 12. Method validation: mass flow rate calculated along the PSHE channel cross section with the aid of PTV. Flow direction is downward.

Mass flow rate computations were also performed at the channel inlet and outlet; see grid volume details in Fig. 5. For these computations, two cylindrical coordinate systems with their origins in the inlet and outlet manifold centers are applied. Mass flow rate integrations in the channel inlet and outlet result in 0.50 and $0.51 \mathrm{~kg} / \mathrm{s}$, respectively.
These values also match the Coriolis measurement within the measurement uncertainty.

To investigate how the velocity vectors are distributed in a given grid point, the probability density functions (PDFs) for the lateral and streamwise velocity components are presented in a central grid location, where the mean lateral velocity should be zero. The grid point $[x=0 ; y=0]$ is chosen for this investigation; see Fig. 4a. Fig. 13 provides the normalized PDFs for lateral (13a) and axial (13b) velocity components in the mentioned grid point.

The PDF of the lateral velocity component is bell-shaped and resembles a Gaussian distribution reasonably well. This is an indication that particle migration in lateral direction caused by any kind of lift force is insignificant. Another indication for the absence of velocity bias in the lateral direction is the agreement between the PTV results for downward and upward flow. The wide probability density function obtained for the vertical component is expected, since the particle velocity is gathered at arbitrary distance from the walls within a grid volume. Similar distributions were observed in other grid points. Note that considerable error-bars in Fig. 12 are due to the variation of velocity over the height.

In order to show that the velocity field measured in the present test section is typical of a commercial PSHE channel, pressure drop measurements were performed in a channel consisting of titanium plates with the same channel inner dimension and at the same Reynolds 


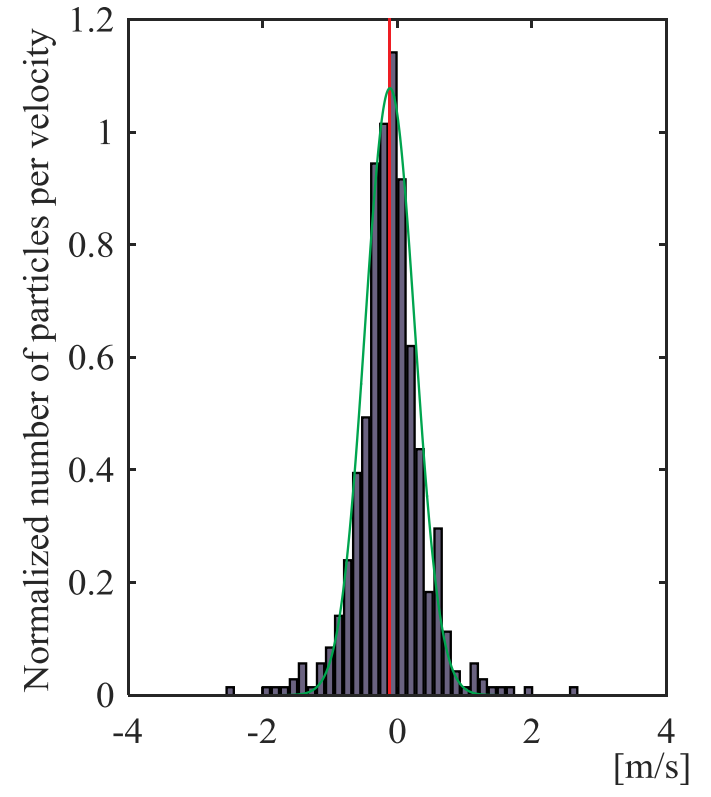

a

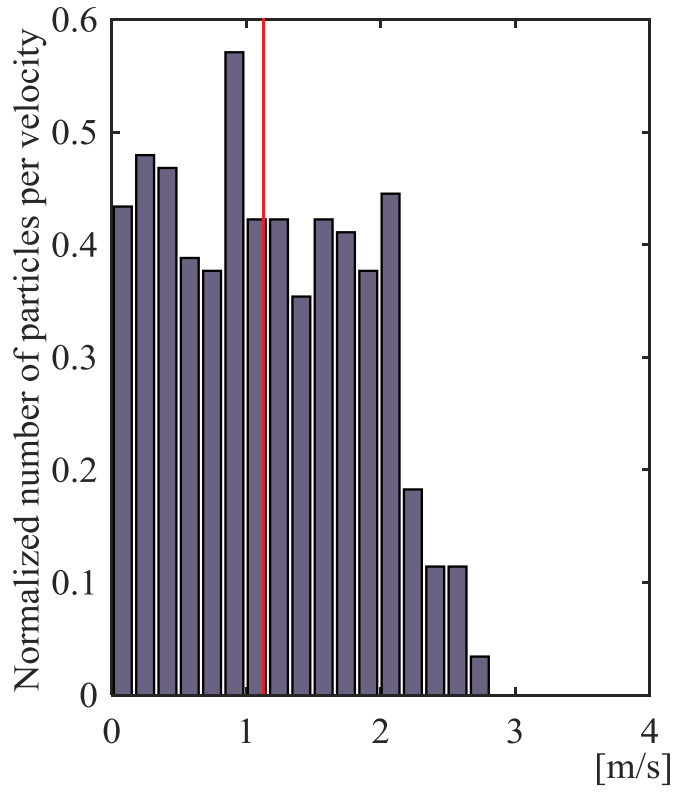

b

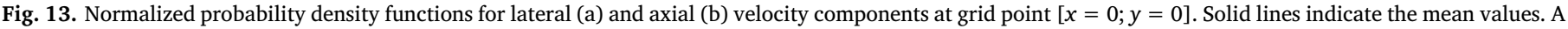
dashed line represents the Gaussian distribution with zero mean and standard deviation equal to $0.37 \mathrm{~m} / \mathrm{s}$.

number. The measured pressure drops are 0.112 and 0.115 bar for the acrylic test section and for the typical PSHE plates, respectively. Although the difference between these results is larger than the measurement uncertainty of the pressure transducer, the difference is small and can be explained by slight differences of the acrylic test section geometry owing to manufacturing limitations.

For the generalization of the present findings it has been verified that the pressure drop can be estimated from friction factor correlations developed for Plate Heat Exchangers (PHE) and applied to the PSHE geometry with the bulk velocity, $v_{\mathrm{B}}$, at the channel mid-plane. The channel Reynolds number is: $R e=\rho\left|v_{\mathrm{B}}\right|(2 b / \phi) / \mu$. It turns out that the pressure drop values computed with the friction correlation by Heavner et al. (1993), $\Delta \mathrm{P}_{\text {Heavner, }}$ differ from measurements, $\Delta \mathrm{P}_{\text {meas }}$, by less than $\pm 5 \%$; see Fig. 14 . The experimental values, $\Delta P_{\text {meas }}$, are presented on the horizontal axis, whereas the vertical axis shows the pressure drop estimations, $\Delta \mathrm{P}_{\text {Heavner}}$.

One can expect reasonable estimations for the PSHE Nusselt number if a similar procedure is applied.

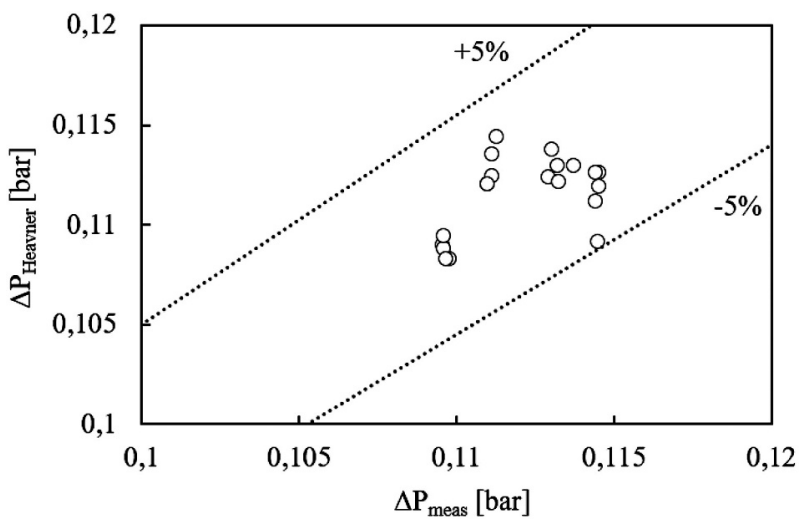

Fig. 14. Effect of Heavner et al. friction factor derived for PHE on PSHE pressure drop estimations.

\section{Conclusions}

Particle trajectories, mean velocities, and estimations of local friction factors, Nusselt numbers and forces due to shear stress have been determined by PTV in a PSHE channel with $15{ }^{\circ}$ Chevron angle at $R e_{b}=3450$ and for flows with ratios of Grashof to Reynolds number squared less than one. Velocity vectors projected onto the channel frontal view derived by straightforward interpolations of consecutive positions of a particle trajectory have been found to yield the mean flow characteristics.

Particle trajectories mostly resemble longitudinal wavy flow (helical type) as presented by Focke and Knibbe (1986) for channels in Plate Heat Exchangers with elevated pressure drops. Particles hardly follow the corrugation angle direction ( $15^{\circ}$ with horizontal). The mean velocity is higher in the central area of the channel, since the flow resistance is smaller there. The mean velocity profile is non-uniform at a given channel cross section. The peak velocity at the channel center is 1.5 times the velocity averaged over the entire mid-plane.

While the fluid enters the PSHE channel on well-behaved streamlines, high turbulence levels and flow recirculation characterize the outlet. Flow recirculation is found that affects the radial velocity distribution. The radial velocity can be as high as 1.20 times the mean velocity averaged over the mid-plane, whereas this ratio below 0.65 at the PSHE entrance. In half of the outlet segment, the radial velocity is close to zero because of the recirculation zone.

It has been shown that the friction factors given by Heavner et al. (1993) when applied to the PSHE geometry with the bulk velocity at the channel mid-plane, predict the measurements well. These equations are apparently useful in generalizing the present findings to other geometries.

The non-uniform velocity field of course affects heat transfer and pressure drop across the PSHE channel. The mean predicted Nusselt number is nearly 76, but it can locally increase up to 110 at the channel center and drop to less than 30 near the channel boundaries. The friction factor as a function of the local Reynolds number yields a 2D map that differs from the corresponding Nusselt maps, since the Nusselt number is inversely proportional to the local Reynolds number. The mean friction factor is 0.135 . It drops to 0.12 at the channel center, 
while it can increase to 0.3 near the PSHE boundaries.

Potential failure locations have been identified. Forces due to shear stresses in horizontal direction are significant only at the inlet and outlet boundaries, whereas forces in vertical direction are significant in the central channel area. Maximum force locations occur at the outlet boundary circumference.

\section{References}

Albrecht, H.-.E., Borys, M., Damaschke, N., Tropea, C., 2003. Laser Doppler and Phase Doppler Measurement Techniques. Springer-Verlag.

Arsenyeva, O.P., Tovazhnyanskyy, L.L., Kapustenko, P.O., Khavin, G.L., Yuzbashyan, A.P., Arsenyev, P.Y., 2016. Two types of welded plate heat exchangers for efficient heat recovery in industry. Appl. Therm. Eng. 105, 763-773.

Cengel, Y.A., 2002. Heat Transfer: A practical Approach, 2nd ed. McGraw-Hill, pp. 487 page.

Clift, R., Grace, K.J.R., Weber, M.E., 1978. Bubbles, Drops and Particles. Academic, New York.

de Boer, A., Oliveira, J.L.G., van der Geld, C.W.M., Malskat, W.S.J., van den Bos, R., Nijsten, T., van Gemert, M.J.C., 2017. Moving heat source in a confined channel: heat transfer and boiling in endovenous laser ablation of varicose veins. Int. J. Heat Mass Transf. 113, 153-165.

Dracos, T., 1996. Particle tracking in three-dimensional space. In: Dracos, T. (Ed.), Three Dimensional Velocity and Vorticity Measuring an Image Analysis Techniques. Kluwer Academic Publishers.

Focke, W., Knibbe, P., 1986. Flow visualization in parallel-plate ducts with corru- gated walls. J. Fluid Mech. 165 (-1), 73-77.

Freire, L.O., de Andrade, D.A., 2014. On applicability of plate and shell heat exchangers for steam generation in naval PWR. Nucl. Eng. Des. 280, 619-627.
Grabenstein, V., Polzin, A.-.E., Kabelac, S., 2017. Experimental investigation of the flow pattern, pressure drop and void fraction of two-phase flow in the corrugated gap of a plate heat exchanger. Int. J. Multiph. Flow 91, 155-169.

Heavner, R.L., Kumar, H., Wanniarachchi, A.S., 1993. Performance of an industrial plate heat exchanger: effect of chevron angle. AIChE Symp. Ser. 89, 262-267.

Klemes, J.J., Arsenyeva, O., Kapustenko, P., Tovazhnyanskyy, L., 2015. Compact Heat Exchangers For Energy Transfer Intensification: Low Grade Heat and Fouling Mitigation. CRC Press, Boca Raton, USA.

Laws, E.M., Lim, E.-H., Livesey, J.L., 1987. Momentum balance in highly distorted turbulent pipe flows. Exp. Fluids 5, 36-42.

Lim, Junyub, Song, Kang Sub, Kim, Dongwoo, Lee, DongChan, Kim, Yongchan, 2018. Condensation heat transfer characteristics of R245fa in a shell and plate heat exchanger for high-temperature heat pumps. Int. J. Heat Mass Transf. 127, 730-739.

Maas, H.-.G., 1996. Contributions of digital photogrammetry to 3D-PTV. In: Dracos, T. (Ed.), Three-Dimensional Velocity and Vorticity Measuring an Image Analysis Techniques. Kluwer Academic Publishers.

Oliveira, J.L.G., van der Geld, C.W.M., Kuerten, J.G.M., 2013. Lagrangian and Eulerian statistics of pipe flows measured with 3D-PTV at moderate and high Reynolds numbers, flow. Turbul. Combust. 91 (1), 105-137.

Oliveira, J.L.G., van der Geld, C.W.M., Kuerten, J.G.M., 2015. Turbulent stresses and particle break-up criteria in particle-laden pipe flows. Int. J. Heat Fluid Flow 53, 44-55.

Oliveira, J.L.G., van der Geld, C.W.M., Kuerten, J.G.M., 2015. Lagrangian velocity an acceleration statistics of fluid and inertial particles measured in pipe flow with 3D particle tracking velocimetry.". Int. J. Multiph. Flow 73, 97-107.

Oliveira, J.L.G., van der Geld, C.W.M., Kuerten, J.G.M., 2017. Concentration and velocity statistics of inertial particles in upward and downward pipe flow. J. Fluid Mech. 822, 640-643.

Walpot, R.J.E., Kuerten, J.G.M., van der Geld, C.W.M., 2006. Experimental determination of Lagrangian velocity statistics in turbulent pipe flow. Flow Turbul. Combust. 76 (2), $163-175$. 